\title{
Recent development and application of a rapid flood spreading method
}

Julien Lhomme, Paul Sayers, Ben Gouldby, Paul Samuels, Martin Wills \& Jonatan Mulet-Marti

Reproduced from:

Flood Risk Management - Research and Practice

Proceedings of FLOODrisk 2008

Keble College, Oxford, UK

30 September to 2 October 2008 


\title{
Recent development and application of a rapid flood spreading method
}

\author{
Julien Lhomme, Paul Sayers, Ben Gouldby, Paul Samuels \& Martin Wills \\ HR Wallingford Ltd., Wallingford, Oxfordshire, UK \\ Jonatan Mulet-Marti \\ Wallingford Software, Wallingford, Oxfordshire, UK (formerly HR Wallingford)
}

\begin{abstract}
Flood risk analysis increasingly involves the integration of a full range of loading conditions as well as multiple defence system states, overlaid by uncertainty analysis. This type of analysis involves the simulation of many thousands of flood events. To keep model runtimes to practical levels an efficient yet robust flood inundation model is required. To accommodate this need a rapid flood spreading model (RFSM) has been developed that utilises the availability of good quality topography data and advanced GIS techniques. This paper describes recent improvements to the RFSM that have focused on incorporating additional physical processes within the spreading algorithm (multiple spilling and friction). This improved model is applied to a number of different sites with comparisons made to a more complex hydrodynamic model. The findings of this comparison demonstrate a good degree of similarity between the RFSM and more complex models, with a significantly reduced runtime overhead.
\end{abstract}

\section{INTRODUCTION}

Flood risk analysis involves the integration of a full range of loading, multiple defence system states and uncertainty related to the input parameters of the model. This type of analysis involves the simulation of many thousands of flood events. To keep model runtimes to practical levels an efficient yet robust flood inundation model is required. To meet these requirements a new model (called RFSM for Rapid Flood Spreading Model) has been developed (Gouldby et al. 2008, HR Wallingford 2006).

This paper provides an overview of the RFSM, the latest developments undertaken and then goes on to compare the latest RFSM to a more complex model on four pilot sites.

\section{OVERVIEW OF THE RFSM CONCEPT}

The RFSM is a simplified hydraulic model that takes as input flood volumes discharged into floodplain areas from breached or overtopped defences (Figure 1). It then spreads the water over the floodplain accounting for the floodplain topography. The output from the model is a flood depth grid of the floodplain area resulting from the input volumes at each defence. The model was specifically developed to provide a fast solution to the flood spreading problem for use in probabilistic flood risk models that consider defence failures (i.e. where many model runs, involving different defence failure combinations, are required).

The pre-process divides the floodplain in elementary areas called Impact Zones (IZs). The IZs represent topographic depressions in the floodplain where the water accumulates in case of flooding (Figure 2). The characteristics of the IZs are also generated by the pre-processing tool (relations between a given IZ and its neighbours, level-volume curve of each IZ). The communication level (CL) of an impact zone defines the level at which water spills into a given neighbour IZ.

The RFSM spreads the flood volumes by filling the IZs adjacent to the input points and spilling the excess to the neighbour IZs. This filling/spilling process is repeated as long as some IZs have volume in excess. When two or more neighbour IZs have the same water level, they are merged into a unique IZ. When all the input volumes have been spread in the IZs and no IZ has excess volume, it is considered that the flood has reached its final state.

This process can be summarized in 5 steps as shown in Figure 3:

- Step 1, the overtopped volume is passed to the IZ adjacent to the defense (IZ B). 


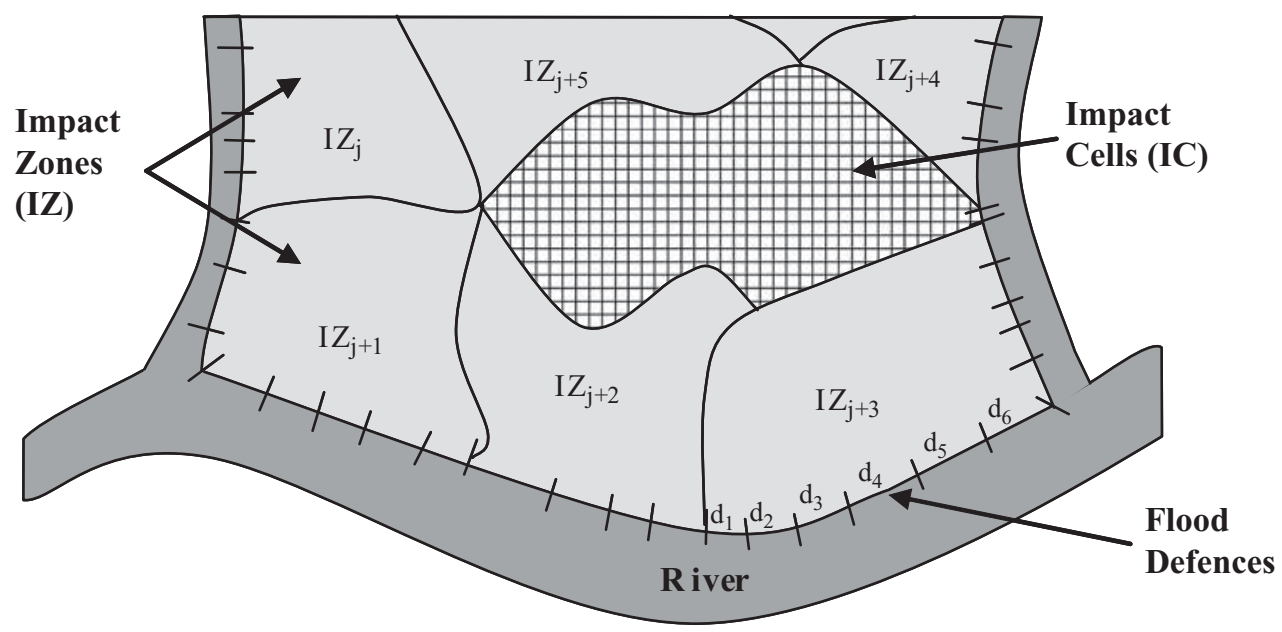

Figure 1. View of the defence system with the Impact Zones and Impact Cells (based on (Gouldby et al. 2008)).

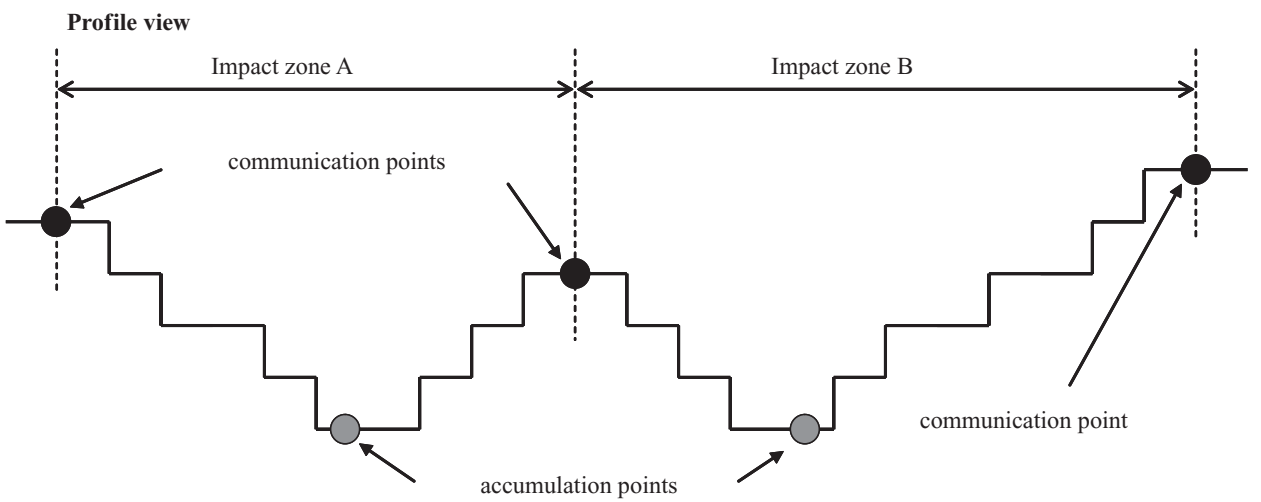

Plan view

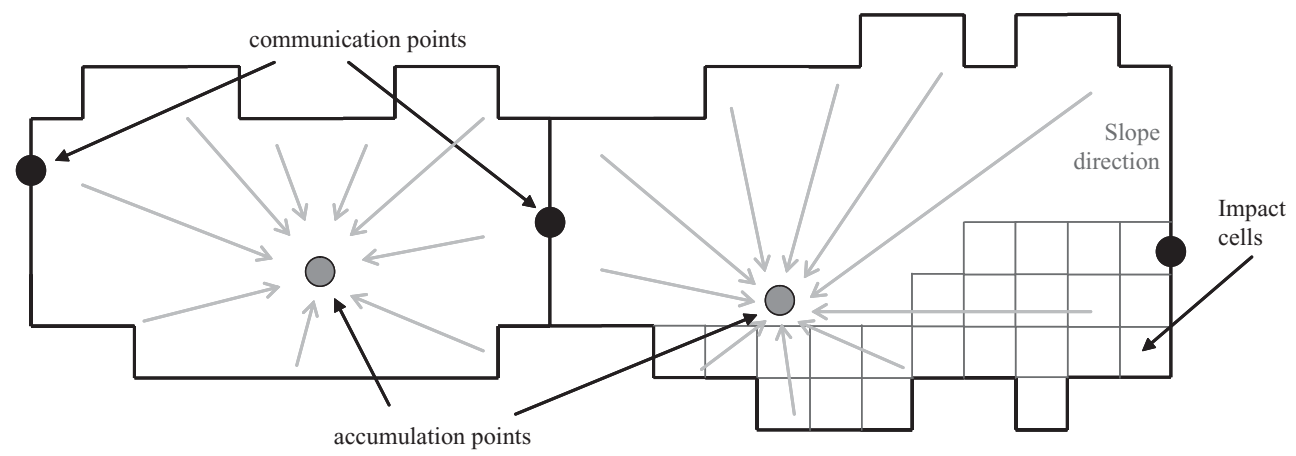

Figure 2. Principles and key features of the Impact Zones (based on (Gouldby et al. 2008)). 


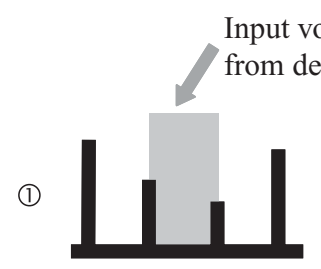

A $\quad$ B $\quad$ C

(3)

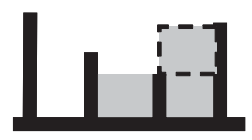

(4)

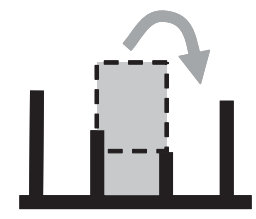

(5)

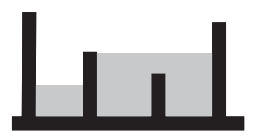

Figure 3. Description of the different spilling/merging steps.

- Step 2, the water level is set to the first CL, this allows to calculate the volume stored in the IZ and the excess volume. The excess volume is spilled towards one or more neighbour IZs (IZ C).

- Step 3, the water level in IZ C being set to the first $\mathrm{CL}$, IZ C has the same water level as IZ B.

- Step 4, IZs B and C are merged. The CLs of this merged IZ (IZ BC) are calculated and the water level is set to the first CL. The excess volume is calculated and spilled towards one or more neighbour IZs (IZ A).

- Step 5, the water volume is lower than the capacity of the IZ and the process stops.

A key feature of the RFSM is the conditions that control the spilling of excess water from one IZ to the next. The next section presents in more detail the spilling conditions and the incorporation of friction influences in addition to the gravitational forces within the RFSM.

\section{DESCRIPTION OF THE MULTIPLE SPILLING AND FRICTION APPROACH}

In the earlier version of the RFSM (Gouldby et al. 2008, HR Wallingford 2006), an IZ with excess volume only spills towards the neighbour IZ with the lowest CL. If two or more neighbour IZs have a CL equal to the lowest CL, the excess volume is shared between them (Figure 4).

In order to improve the RFSM, it was recognized that the algorithm should incorporate more physical processes, like the dynamic effects during the filling of an IZ and the friction effects during the spreading.
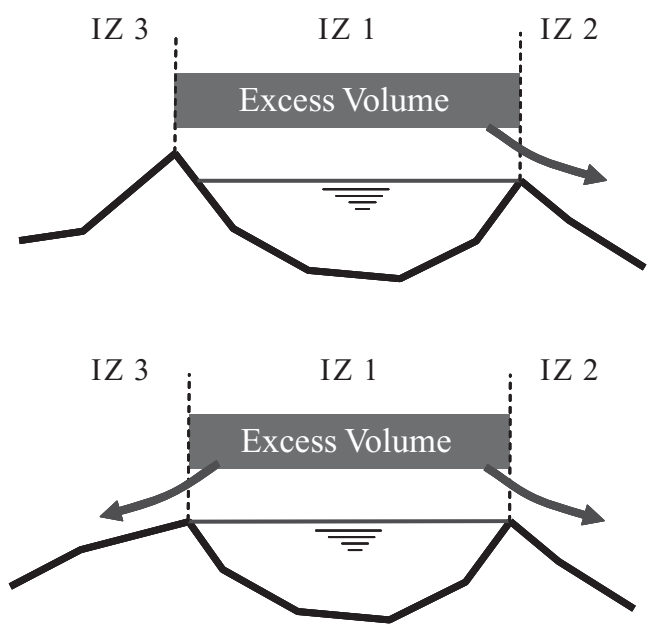

Figure 4. Description of the spilling rules in the earlier RFSM.

\subsection{Multiple spilling}

The approach chosen to represent the dynamic effects during the filling has been called Multiple Spilling. This can be achieved in two different ways :

i. changing the CLs during the pre-processing step so the CLs that are similar are set as equal, allowing any excess volume to be shared between them;

ii. modifying the spilling algorithm so that the excess volume can be spilled towards multiple neighbours by taking account of the relative elevation of the CLs.

The first approach was used in the original version of the RFSM, this was done by introducing a Tolerance parameter. The tolerance parameter allows CLs that are similar (where similar is defined using the tolerance parameter) to be considered as equal and the excess volume shared between them.

This was implemented within the pre-processing routine by resetting all CLs between $n \times$ Tolerance and $(n+1) \times$ Tolerance to $(n+1 / 2) \times$ Tolerance with $n$ an integer. If the Tolerance is set to zero, no adjustment is done.

The main drawback of this approach is that it lacks flexibility in the adjustment of the CLs. Considering a Tolerance parameter equal to $0.2 \mathrm{~m}$ and $n$ equal to 3 , two CLs respectively equal to 0.59 and $0.61 \mathrm{~m}$ will be adjusted to different values (respectively 0.50 and $0.70 \mathrm{~m}$ ) although they are very close.

The second approach has been implemented in the latest version of the RFSM to have a better representation of the spilling. Instead of using a constant tolerance applied to all CLs between any 
neighbour IZs, the tolerance is calculated for each IZ as a function of its shape. The functional relationship between the tolerance parameter and the IZ shape is explained below:

Consider the shape of the IZ as approximated by a cone. If the cone has a wide aperture (Figure 5a), the water level in the impact zone will increase relatively slowly for any given input discharge. Conversely, if the cone has a small aperture (Figure 5b), the water level in the IZ will increase rapidly for the same input discharge. It is more likely that there will be spilling towards many neighbour IZs if the water level is rising rapidly.

The nature of the impact zone is captured within the RFSM through the Volume-Level relation (Figure 6). IZ 1 has a higher average Volume-Level slope than IZ 2, despite both having approximately the same capacity. Thus for a given input volume the level will increase more rapidly in IZ 1 than in IZ 2. So IZ 1 and IZ 2 can respectively be associated with the shapes in Figure $5 \mathrm{a}$ and Figure $5 \mathrm{~b}$.

The tolerance for each IZ is then calculated using the model variable, referred to as MSTol (for Multiple Spilling Tolerance), as follows:

MSTol $=K T o l \times \frac{\text { Level }_{2}-\text { Level }_{1}}{\text { Volume }_{2}}$

where Level $_{2}$ and Level $_{1}$ are respectively the elevations of the first CL and the lowest point in the IZ (i.e. a notional depth of the IZ), Volume 2 is the volume of water in the IZ when the water level reaches Level $_{2}$, $K T o l$ is a constant parameter.
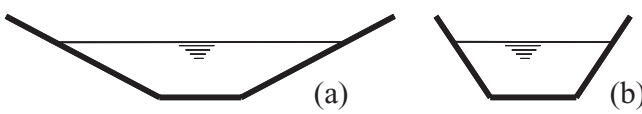

Figure 5. Link between the IZ shape and the dynamic filling effects.

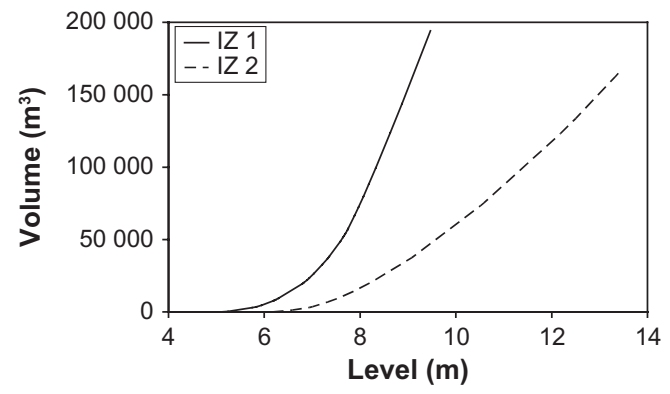

Figure 6. Example of two volume-level curves.
MSTol is considered as an additional depth of water in the IZ due to the dynamic effects during the filling. In reality the excess volume will be discharged in the first neighbour IZ over a certain time allowing the water level to rise above the first CL and then possibly reach the second CL. MSTol is calculated and applied by the analysis module (rather than by the pre-processing). This also avoids any modification of the CL and hence the CLs used in the RFSM remain a true representation of the DTM (see section 3.3).

To explore the sensitivity of the RFSM to the introduction of the MSTol parameter and in particular its sensitivity to the constant KTol, a significant number of sensitivity tests have been undertaken. These tests show that the RFSM is relatively insensitive to KTol and a default value of 1400 for KTol provides satisfactory results for all tested situations (when used in conjunction with the friction effects, see next section).

\subsection{Friction}

An important process for floodplain flow models is surface friction. In particular, floodplain land cover causes friction which affects the movement of the flood wave. Typically a Manning friction coefficient is used to capture this physical effect. The original RFSM made no allowance for friction, this was recognized as a significant omission in the case of low lying coastal floodplains where the flood extents were too large.

This short-coming has been addressed by linking the influence of the friction effect to the plan size of the flooded area. The larger the flooded area, the greater the friction effects are.

The friction effects are represented through a head-loss. This head-loss is added to the CL to give the threshold value that the water level needs to exceed before spilling (see section 3.3). This head-loss is considered to be a function of the total wet area by the linear relationship:

$S_{f}=C_{f} \times A_{\text {total }}$

where $S_{f}$ is the friction head-loss $(\mathrm{m}), A_{\text {total }}$ is the flooded area $\left(\mathrm{m}^{2}\right), C_{f}$ is a constant coefficient $\left(\mathrm{m}^{-1}\right)$.

The friction head-loss is calculated for every iteration (an iteration being a single spilling/merging step) as the flooded area varies after each iteration. The friction head-loss is applied for spilling only towards empty IZs.

The sensitivity of the RFSM to the parameter $C_{f}$ has been examined. Although the RFSM is relatively sensitive to the parameter $C_{f}$, the value $10^{-9}$ has been found giving satisfactory results for all tested situations (when used in conjunction with the multiple spilling). 

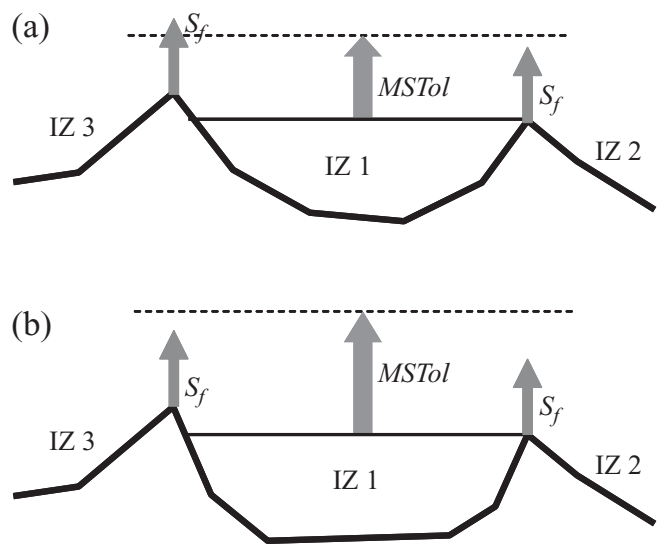

Figure 7. Description of the spilling rules in the latest version of RFSM, with the combined role of multiple spilling $(M S T o l)$ and friction $\left(S_{f}\right)$.

\subsection{Overview of the spilling algorithm}

The condition for spilling in the earlier RFSM was as follows:

$$
\text { WaterLevel }=C L_{1}
$$

where CL1 is the lowest CL. This has been modified in the latest RFSM with the introduction of the multiple spilling and friction effects. The spilling condition becomes:

WaterLevel $+M S T o l \geq C L+S_{f}$

where the water level is set to the first CL.

These conditions are explained in Figure 7. In Figure 7a, the sum of the water level and MSTol is higher than $\mathrm{CL}_{\mathrm{IZ1} 1 \mathrm{IZ} 2}+S_{f}$, but lower than $\mathrm{CL}_{\mathrm{IZ1-IZ3}}+S_{f}$ Then the spilling of the excess volume is done only towards IZ 2. In Figure 7b, the sum of the water level and MSTol is higher than CL $+S_{f}$ for both neighbour IZs. So the spilling is done towards both IZs 2 and 3.

\section{APPLICATION ON PILOT SITES}

\subsection{Method}

The RFSM performance has been evaluated over 4 pilot sites through a comparison with the $2 \mathrm{D}$ hydrodynamic model Tuflow. The RFSM used here is the latest version with multiple spilling and friction.

The final flood depths computed by Tuflow are here considered to be the correct results and are used to calculate the RFSM performance indicators.
The performance indicators used here are:

- the mean deviation between the RFSM depths and the Tuflow depths (referred below as mean deviation);

- a fit indicator and a bias indicator to quantify the matching of the flood extent from both models (referred below as fit and bias).

The fit and bias are calculated using the following relations

$$
\begin{aligned}
& \text { Fit }=100 \times \frac{B}{B+C+D} \\
& \text { Bias }=100 \times\left(\frac{B+C}{B+D}-1\right)
\end{aligned}
$$

where $B$ is the area wet in both models, $C$ is the area wet in RFSM but dry in Tuflow, $D$ is the area wet in Tuflow but dry in RFSM, $A$ being the area dry in both models (Bates and De Roo, 2000).

\subsection{Carlisle, Cumbria, UK}

The first pilot site is located in the city centre of Carlisle (UK). A filtered LiDAR data with a grid size of $10 \mathrm{~m}$ has been used in both models. The area is described by 72,580 DTM cells $\left(7.2 \mathrm{~km}^{2}\right)$. The RFSM pre-processing allows to reduce the number of elements to only 3227 IZs.

18 input points have been defined and a triangular hydrograph is injected in the model at every point. The total input volume is $7.9710^{6} \mathrm{~m}^{3}$. The Tuflow model is a $2 \mathrm{D}$ only model with the input points being exactly the same as in the RFSM. The Tuflow model runs in approximately $20 \mathrm{mn}$, whereas the RFSM runs in only $2 \mathrm{~s}$.

Both models give very similar flood extent and flood depths. This is due to the high importance of the topography in this constrained floodplain. The mean depth deviation over the whole area is $0.06 \mathrm{~m}$, the fit and bias are respectively $98.4 \%$ and $1.2 \%$. The comparison of the flood extent is shown in Figure 8.

\subsection{River Brit, Dorset, UK}

The second pilot site is a reach of the River Brit at Bridport. The area is described by a $5 \mathrm{~m}$ DTM comprising 145,905 cells $\left(3.6 \mathrm{~km}^{2}\right)$. The RFSM pre-processing reduces the number of elements to $5702 \mathrm{IZs}$.

A coupled 1D/2D Tuflow model of the river and floodplain has been used to estimate the volumes spilled into the floodplain. In the RFSM the spills from the river are represented by 37 input points with 


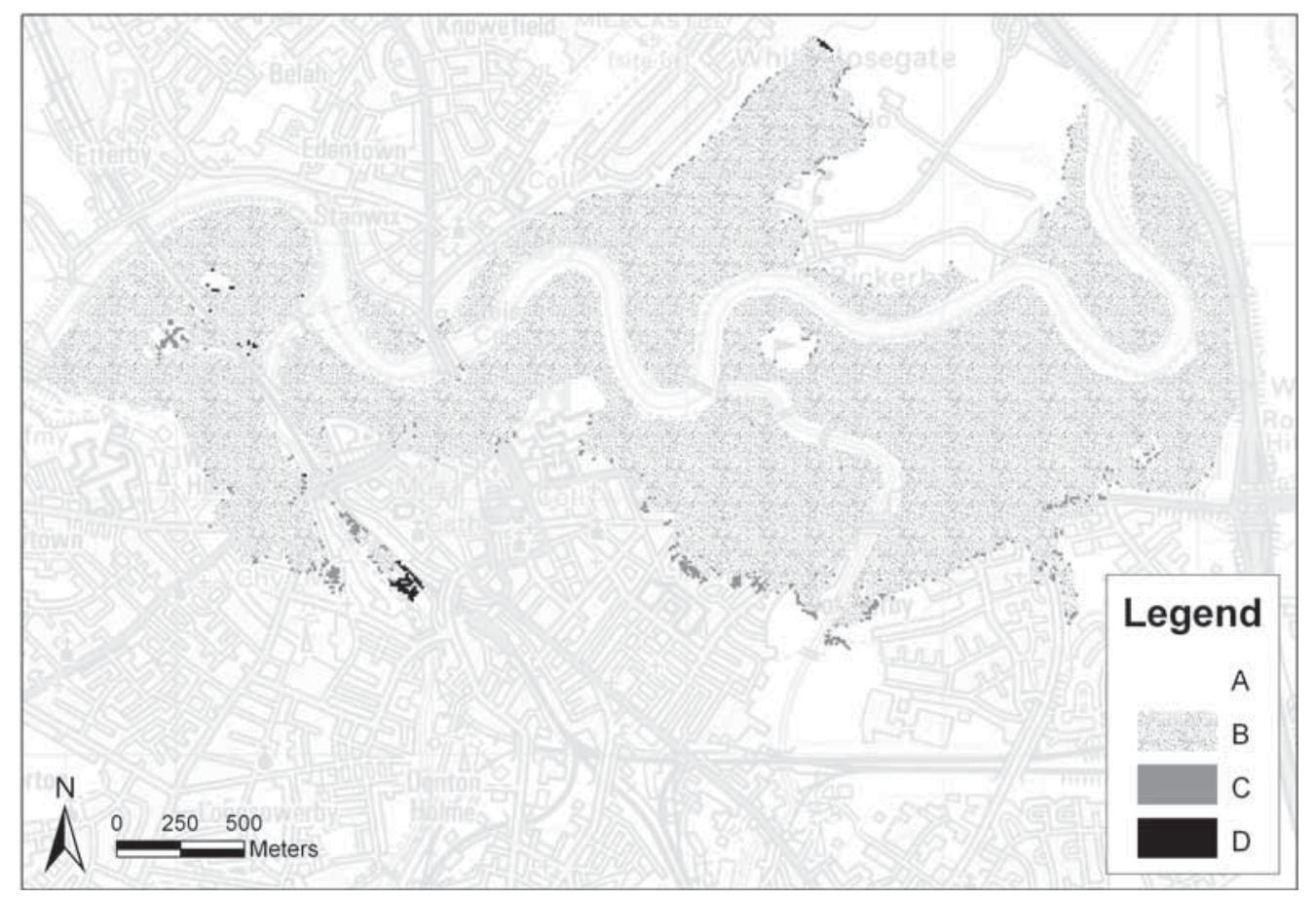

Figure 8. Comparison of flood extent from RFSM and Tuflow in Carlisle.

a total input volume of $804,900 \mathrm{~m}^{3}$. The Tuflow model runs in $11 \mathrm{~h}$ whereas the RFSM runs in $4 \mathrm{~s}$.

The two model outputs are similar in the downstream part of the site, but the differences are higher in the upstream part. This is partly explained by the limited number of input points in RFSM. The mean depth deviation over the whole area is $-0.06 \mathrm{~m}$, the fit and bias are respectively $81.8 \%$ and $-7.7 \%$. The comparison of the flood extent is shown in Figure 9.

\subsection{Boston, East Anglia, UK}

The third pilot site is a large flat coastal floodplain in the area of Boston (UK). The site is described by a $50 \mathrm{~m}$ grid mixed Lidar/SAR DTM comprising 387,265 cells $\left(968 \mathrm{~km}^{2}\right)$. The RFSM pre-processing reduces the number of elements to $28,609 \mathrm{IZs}$.

A RASP system risk model (Gouldby et al. 2008) had been previously applied and established a set of overtop/breaching volumes for a number of return periods and for each defence (HR Wallingford, 2007). For the testing of the revised RFSM a nominal 1000 year storm event is considered with a combination of breaches and overtopping.

The corresponding volumes are injected in the Tuflow model ( $2 \mathrm{D}$ domain only) at 46 points. The
Tuflow model runs in $15 \mathrm{~h}$ whereas the RFSM runs in $5 \mathrm{~s}$.

The mean depth deviation over the whole area is approximately $0.04 \mathrm{~m}$, the fit and bias are respectively $56.7 \%$ and $4.1 \%$. The comparison of the flood extent is shown in Figure 10. The RFSM does not perform as well as in the previous sites in term of flood extent. This is largely due to the flat topography giving more importance to the inertial terms in the shallow water equations which are not currently represented in the RFSM.

However the inclusion of the multiple spilling and friction terms in RFSM considerably improves the flood extent compared to the original version of RFSM (shown in Figure 11).

\subsection{River Lee, London, UK}

The fourth pilot site is a small densely urbanised area in the North East of London. The area is described by a $5 \mathrm{~m}$ Lidar DTM comprising 183,362 cells $\left(4.6 \mathrm{~km}^{2}\right)$. The RFSM pre-processing reduces the number of elements to $6788 \mathrm{IZs}$.

The considered flood event is caused by flows from a surcharging culvert and is modeled by two input hydrographs. The total input volume is $506,400 \mathrm{~m}^{3}$. 


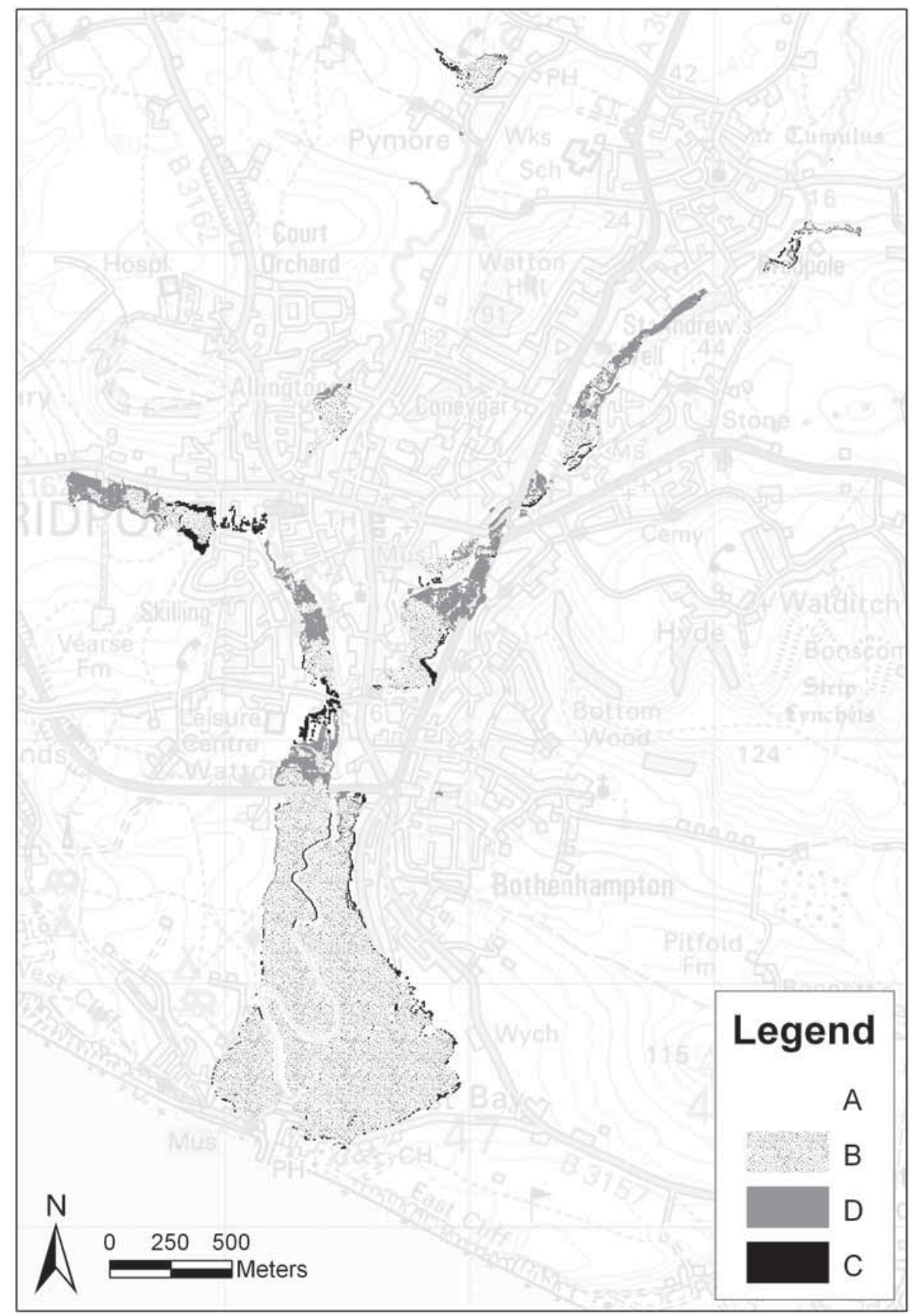

Figure 9. Comparison of flood extent from RFSM and Tuflow on the River Brit. 


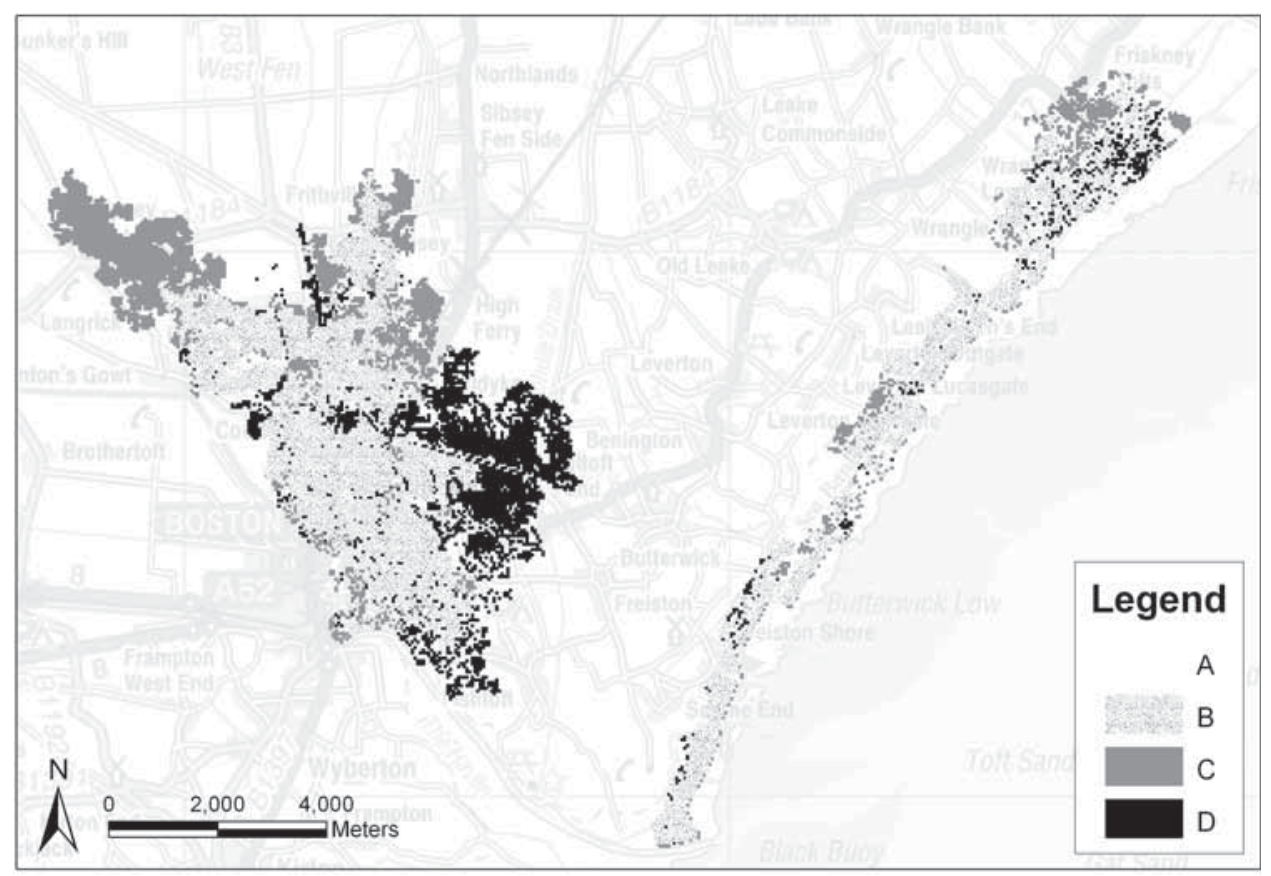

Figure 10. Comparison of flood extent from RFSM and Tuflow in Boston (latest version of RFSM).

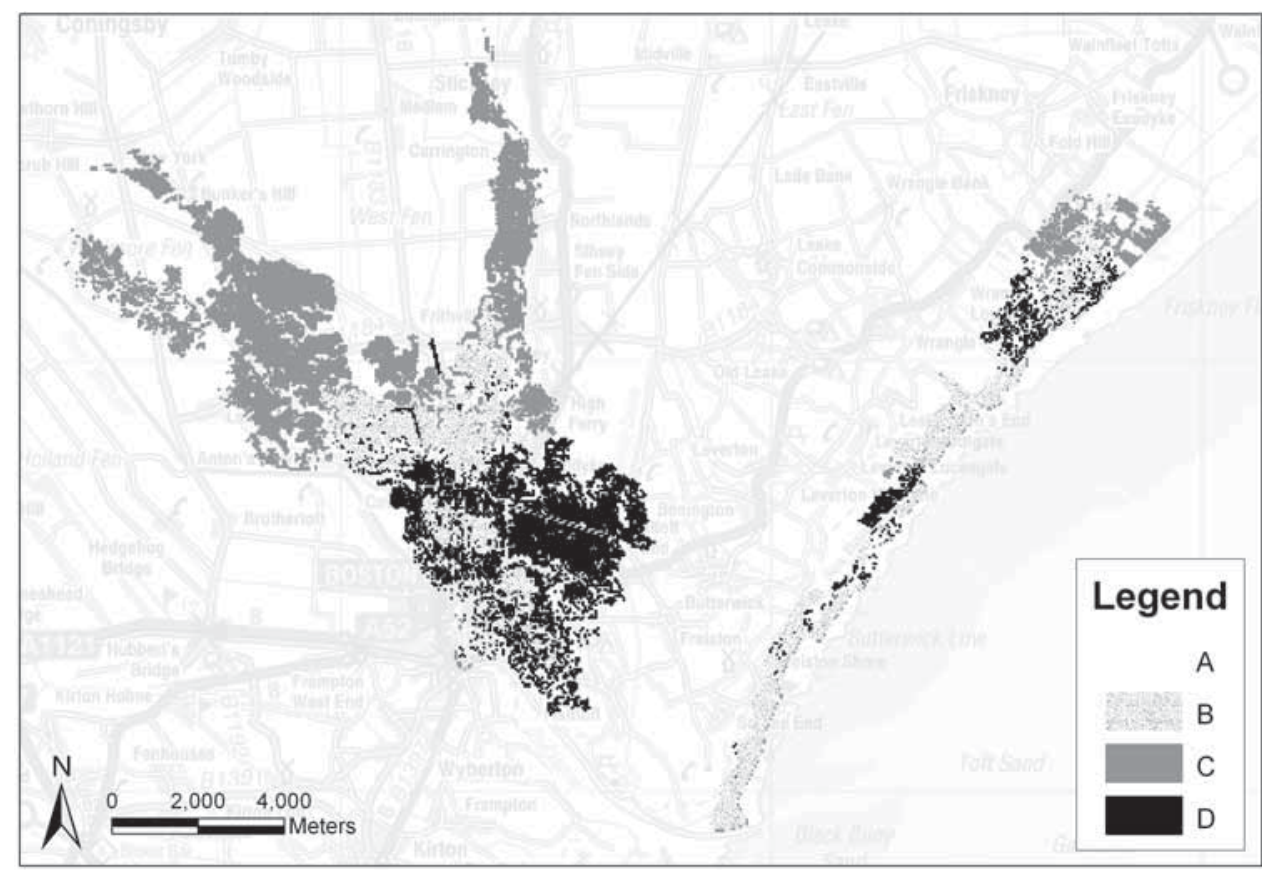

Figure 11. Comparison of flood extent from RFSM and Tuflow in Boston (original version of RFSM). 


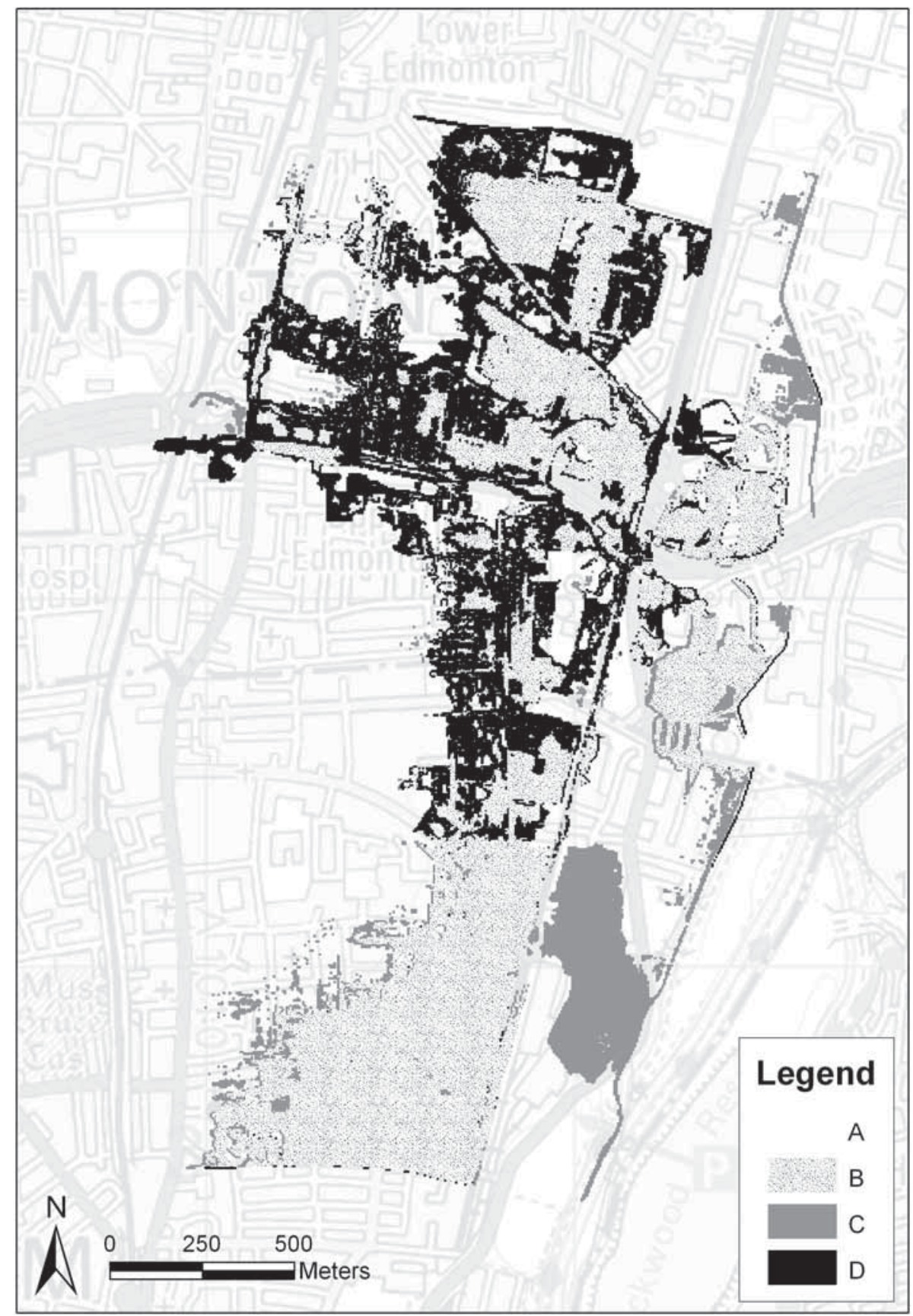

Figure 12. Comparison of flood extent from RFSM and Tuflow on the River Lee. 
Table 1. Summary of the comparison indicators between RFSM and Tuflow for the 4 pilot sites.

\begin{tabular}{lllr}
\hline Pilot site & $\begin{array}{l}\text { Mean depth } \\
\text { deviation }(\mathrm{m})\end{array}$ & $\begin{array}{l}\text { Fit } \\
(\%)\end{array}$ & $\begin{array}{l}\text { Bias } \\
(\%)\end{array}$ \\
\hline Carlisle & +0.06 & 98.4 & +1.2 \\
River Brit & -0.06 & 81.8 & -7.7 \\
Boston & +0.04 & 56.7 & +4.1 \\
River Lee & +0.07 & 54.3 & -23.6 \\
\hline
\end{tabular}

The Tuflow model runs in $2 \mathrm{~h}$ approximately whereas the RFSM runs in 10s.

The mean depth deviation over the whole area is approximately $0.07 \mathrm{~m}$, the fit and bias are respectively $54.3 \%$ and $-23.6 \%$. The comparison of the final depths in both models is shown in Figure 12.

\section{CONCLUSIONS}

The RFSM is a flood spreading model designed to run with very short computational and setup times. This type of model is directly suited:

- To system risk analysis (where many flood inundation realisations are required to represent a full range of loading, multiple defence system states and associated uncertainty.

- To large geographic scales where model set up should be efficient and run time short.

The RFSM has been shown to perform well against Tuflow in a number of pilot sites (Table 1). It is much faster to run than hydrodynamic model (run time typically $<5 \mathrm{~s}$ ) and appropriately accurate.
The incorporation of multiple spilling and friction effects in the algorithm are clearly improving the model outputs in flat floodplains.

The RFSM continues to be developed in terms of both the pre-processing and the spreading algorithms. These developments will support the analysis of impacts within the flood pathway and broader impacts such as risk to life that rely on velocity and duration terms.

\section{ACKNOWLEDGMENTS}

The authors are grateful to the Environment Agency for providing the pilot sites data in Carlisle and River Brit and to Matt Horritt (Halcrow) for providing the Tuflow flood depths on the River Lee pilot site.

Part of this work was done under the Flood Risk Management Research Consortium, funded by the Engineering and Physical Sciences Research Council and the Environment Agency.

\section{REFERENCES}

Bates P., De Roo A. (2000). A simple raster-based model for flood inundation simulation. Journal of Hydrology, Volume 236, pp. 54-77.

Gouldby B., Sayers P., Mulet-Marti J., Hassan M and Benwell D (2008). A methodology for regional-scale flood risk assessment. Proceedings of the Institution of Civil Engineers, Water Management, Volume 161, Issue WM3, pp. 169-182.

HR Wallingford (2006), Rapid Flood Spreading Methodology (RFSM), Environment Agency, Thames Estuary 2100 Report, DT4.

HR Wallingford (2007). Boston SFRA, RASP Model, HR Wallingford, Report EX5511. 


\title{
Fluid thinking...smart solutions
}

HR Wallingford provides world-leading analysis, advice and support in engineering and environmental hydraulics, and in the management of water and the water environment. Created as the Hydraulics Research Station of the UK Government in 1947, the Company became a private entity in 1982, and has since operated as a independent, non profit distributing firm committed to building knowledge and solving problems, expertly and appropriately.

Today, HR Wallingford has a 50 year track record of achievement in applied research and consultancy, and a unique mix of know-how, assets and facilities, including state of the art physical modelling laboratories, a full range of computational modelling tools, and above all, expert staff with world-renowned skills and experience.

The Company has a pedigree of excellence and a tradition of innovation, which it sustains by re-investing profits from operations into programmes of strategic research and development designed to keep it - and its clients and partners - at the leading edge.

Headquartered in the UK, HR Wallingford reaches clients and partners globally through a network of offices, agents and alliances around the world.

\section{HR Wallingford}

Working with water

\author{
HR Wallingford Ltd \\ Howbery Park \\ Wallingford \\ 0xfordshire 0X10 8BA \\ UK \\ $\begin{array}{ll}\text { tel } & +44(0) 1491835381 \\ \text { fax } & +44(0) 1491832233 \\ \text { email } & \text { info@hrwallingford.co.uk }\end{array}$
}

www.hrwallingford.co.uk 